\title{
High-Frequency Sea Level Variations Observed by GPS Buoys Using Precise Point Positioning Technique
}

\author{
Chung-Yen Kuo ${ }^{1, *}$, Kuan-Wei Chiu ${ }^{1}$, Kai-Wei Chiang ${ }^{1}$, Kai-Chien Cheng ${ }^{2}$, Li-Ching Lin ${ }^{3}$, \\ Hong-Zeng Tseng ${ }^{1}$, Feng-Yu Chu ${ }^{1}$, Wen-Hau Lan ${ }^{1}$, and Hsiang-Tseng Lin ${ }^{4}$ \\ ${ }^{1}$ Department of Geomatics, National Cheng-Kung University, Tainan, Taiwan \\ ${ }^{2}$ Department of Earth and Environmental Sciences, National Chung Cheng University, Chiayi, Taiwan \\ ${ }^{3}$ Research Center for Environmental Changes, Academia Sinica, Taipei, Taiwan \\ ${ }^{4}$ Material Production Center, Armaments Bureau, Ministry of National Defense, Taichung, Taiwan
}

Received 11 July 2011, accepted 5 October 2011

\begin{abstract}
In this study, sea level variation observed by a 1-Hz Global Positioning System (GPS) buoy system is verified by comparing with tide gauge records and is decomposed to reveal high-frequency signals that cannot be detected from 6-minute tide gauge records. Compared to tide gauges traditionally used to monitor sea level changes and affected by land motion, GPS buoys provide high-frequency geocentric measurements of sea level variations. Data from five GPS buoy campaigns near a tide gauge at Anping, Tainan, Taiwan, were processed using the Precise Point Positioning (PPP) technique with four different satellite orbit products from the International GNSS Service (IGS). The GPS buoy data were also processed by a differential GPS (DGPS) method that needs an additional GPS receiver as a reference station and the accuracy of the solution depends on the baseline length. The computation shows the average Root Mean Square Error (RMSE) difference of the GPS buoy using DGPS and tide gauge records is around $3-5 \mathrm{~cm}$. When using the aforementioned IGS orbit products for the buoy derived by PPP, its average RMSE differences are $5-8 \mathrm{~cm}, 8-13 \mathrm{~cm}$, decimeter level, and decimeter-meter level, respectively, so the accuracy of the solution derived by PPP highly depends on the accuracy of IGS orbit products. Therefore, the result indicates that the accuracy of a GPS buoy using PPP has the potential to measure the sea surface variations to several cm. Finally, highfrequency sea level signals with periods of a few seconds to a day can be successfully detected in GPS buoy observations using the Ensemble Empirical Mode Decomposition (EMD) method and are identified as waves, meteotsunamis, and tides.
\end{abstract}

Key words: GPS buoy, Precise point positioning, Tide gauge, High-frequency sea level variation

Citation: Kuo, C. Y., K. W. Chiu, K. W. Chiang, K. C. Cheng, L. C. Lin, H. Z. Tseng, F. Y. Chu, W. H. Lan, and H. T. Lin, 2012: High-frequency sea level variations observed by GPS buoys using precise point positioning technique. Terr. Atmos. Ocean. Sci., 23, 209-218, doi: 10.3319/TAO.2011.10.05.01(Oc)

\section{INTRODUCTION}

Quantifying sea level change is desirable because sea level change has a direct impact on societal well-being on a scale of time over decades and centuries. To accurately determine sea level variations is a complex problem. Nowadays the most commonly used instruments to monitor sea level are tide gauges and satellite altimetry (Willis et al. 2010). However, tide gauges are crust-fixed instruments resulting in measuring only relative sea level and do not represent the true sea level signal separating vertical land motion. Sea surface heights from satellite altimeters in

\footnotetext{
* Corresponding author

E-mail:kuo70@mail.ncku.edu.tw
}

coastal regions, on the other hand, are inaccurate because of unreliable geophysical corrections and noisy returned radar waveforms (Lee et al. 2010). In addition, because of data sampling rates, high-frequency sea level variations cannot be sufficiently detected from 6-minute tide gauges measurements or weekly sampled satellite altimetry data. Highfrequency $1-\mathrm{Hz}$ sea level observations from the Global Positioning System (GPS) buoys have the potential to mitigate the drawbacks found with other techniques.

Floating GPS buoys equipped with geodetic-grade dual-frequency GPS antennas have demonstrated the method to be an effective and economical procedure to collect water level readings to monitor sea level variations (Cardellach et al. 2000), calibrate satellite altimetry measurements (e.g., 
Cheng et al. 2010), validate large river water storage catchments (Frappart et al. 2006; Cheng et al. 2009), measure regional geoid (Del Cogliano et al. 2007), and accurately link shoreline vertical datum (Cheng et al. 2008). The principal component of this instrument is the GPS system constellation, which is a space-based global navigation satellite system operated by the US Department of Defense. The basic concept originates from a single point positioning (SPP) method using a single GPS receiver but subject to systemic errors including atmospheric delays, satellite orbit, satellite clock, and receiver clock errors, as well as a multipath effect, and receiver noise. In order to reduce system errors, the previous studies mentioned above and others have demonstrated a cm-level accuracy with a traditional differential GPS (DGPS) technique.

Using the DGPS to determine a buoy's location, an additional receiver with a given coordinate is required to simultaneously collect data and to form differential observation equations in order to reduce significant system errors. As a result, the relative position vector is obtained, instead of the absolute coordinates of the rover. The use of DGPS assumes that both ends of a baseline have similar and highly correlated conditions in ionospheric, tropospheric and other error sources. Hence a DGPS procedure would reduce such errors significantly. However, as the length of a baseline increases, the errors sources are different at the ends of a baseline, so this assumption for reducing errors using DGPS becomes invalid; therefore, the positioning accuracy of a buoy is limited by the baseline length, meaning that GPS buoys cannot be too far from land; a long baseline therefore, becomes corrupted at both ends of the baseline and decreases the accuracy of relative positioning. Consequently, precise point positioning (PPP) technique was developed in an effort to solve this problem.

Zumberge et al. (1997) developed the PPP technique, wherein the fundamental principle uses a zero-differenced dual-frequency code and carrier phase measurements, models to reduce system errors, and precise ephemerides and precise clock corrections. Because absolute and precise [3D RMS kinematic: < $1 \mathrm{~m}$; static: < $0.1 \mathrm{~m}$ ( $\mathrm{Li}$ et al. 2009)] positioning can be obtained with one single receiver, the PPP method has been widely applied to many disciplines of research; for example, Zhang and Andersen (2006) successfully measured the surface ice flow velocity and the tide retrieval of the Amery ice shelf with a GPS using PPP instead of a DGPS method.

Chen et al. (2004) estimated the coordinates of GPS buoys using precise ephemerides and satellite clock error data of the International GNSS Service (IGS) rapid product, whose latency is 17 hours, with kinematic PPP technique and found that the root mean square error (RMSE) is around $10-20 \mathrm{~cm}$ compared to the positioning solution derived by DGPS. However, there is no independent data to absolutely evaluate a GPS buoy solution like tide gauge records.
In this study, we assessed the accuracy of GPS buoy positioning processed by PPP and DGPS techniques with a goal to see if a GPS buoy can be used to measure sea level variation data to obtain high-frequency ocean signals. The kinematic positions of a GPS buoy used in five campaigns were computed by both DGPS and PPP methods using all four kinds of IGS precise ephemerides: the final, the rapid, and the ultra-rapid products (the ultra-rapid product includes the observed half and the predicted half). In addition to the comparison of results derived by PPP and DGPS, the GPS buoy solutions were also compared to a tide gauge record at 6-minute intervals at Anping, Tainan, Taiwan, which is near the deployed GPS buoy. Finally, the high-frequency GPS buoy observed sea level time series were subjected to an examination using the empirical mode decomposition (EMD) method in order to analyze ocean signals.

\section{INTRODUCTION OF GPS BUOY AND PPP TECHNIQUE}

\subsection{GPS Buoy}

GPS buoy is a robust instrument which will continually collect high samples of sea surface heights. When collecting sea surface heights using GPS buoys, the arrangement of GPS antennas is critical. The antenna has to be fixed to the float, and the distance between the phase center and water surface line must be carefully measured (Cheng 2005). Figure 1 shows the structure of the GPS buoy used in this study, which consists of a dual-frequency geodetic-grade GPS receiver. Fortunately, the GPS buoy system is simply constructed at a low price.

\subsection{PPP Technique}

In general, the PPP technique can be used to overcome the limitations of SPP and DGPS methods. If using a dualfrequency GPS receiver, zero-differenced dual-frequency

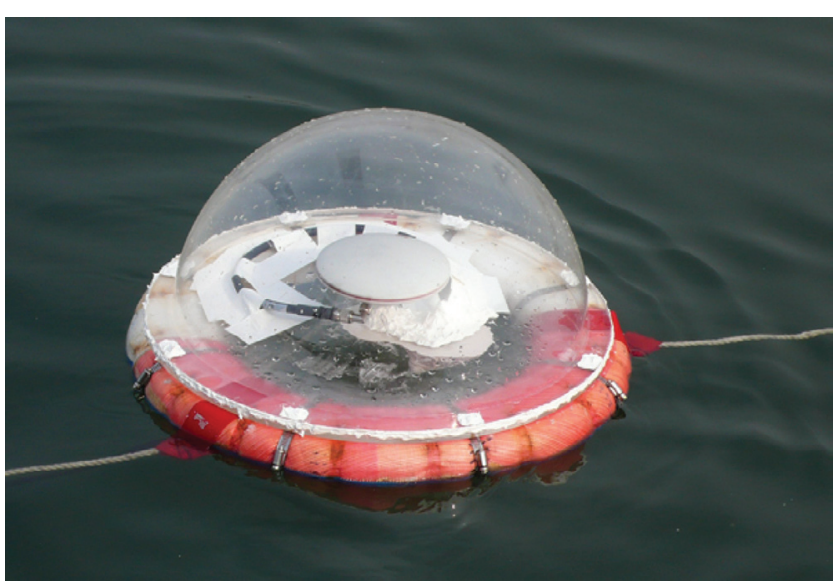

Fig. 1. GPS buoy used in this study. 
code and carrier phase measurements can form an ionosphere-free combination, called the traditional PPP model (Héroux et al. 2001). The equations are as follows:

$$
\begin{aligned}
P_{I F}= & {\left[f_{1}^{2} P(L 1)-f_{2}^{2} P(L 2)\right] /\left(f_{1}^{2}-f_{2}^{2}\right)=\rho+c(d t-d T)+d_{o r b} } \\
& +d_{\text {trop }}+d_{\text {mult } t P(L 1+L 2)}+\varepsilon[P(L 1+L 2)] \\
\Phi_{I F}= & {\left[f_{1}^{2} \Phi(L 1)-f_{2}^{2} \Phi(L 2)\right] /\left(f_{1}^{2}-f_{2}^{2}\right)=\rho+c(d t-d T)+d_{\text {orb }} } \\
& +d_{\text {trop }}+\left(c f_{1} N_{1}-c f_{2} N_{2}\right) /\left(f_{1}^{2}-f_{2}^{2}\right)+d_{\text {mult } \Phi(L 1+L 2)} \\
& +\varepsilon[\Phi(L 1+L 2)]
\end{aligned}
$$

where $P(L i)$ is the $L i$ pseudorange; $L i$ is the carrier with frequency $f_{i} ; \Phi(L i)$ is the carrier phase; $\rho$ is the true geometric distance between a satellite and the receiver; $c$ is the speed of light in vacuum; $d t$ is the satellite clock error; $d T$ is the receiver clock error; $d_{o r b}$ is the satellite orbit error; $d_{t r o p}$ is the tropospheric delay; $N_{i}$ is the non-integer ambiguity of carriers; $d_{m u l t / P(L i)}$ and $d_{m u l t} \Phi(L i)$ are the multipath effects; and $\varepsilon[P(L i)]$ and $\varepsilon[\Phi(L i)]$ are other sources of noise.

However, there are still two problems: no characteristic of integer ambiguity and significantly amplified noise. Gao et al. (2003) and Abdel-Salam et al. (2002) solved the problems by smoothing the pseudo-ranges and then averaging the pseudo-ranges with carrier phase observations. The equations are expressed as:

$$
\begin{aligned}
P_{I F, L i}=0.5[ & \left.P_{S M}(L i)+\Phi(L i)\right] \\
P_{S M, L i}(n)= & \frac{1}{m} P_{L i}(n)+\left(1-\frac{1}{m}\right)\left[P_{S M, L i}(n-1)+\Phi_{L i}(n)\right. \\
& \left.-\Phi_{L i}(n-1)\right]=\rho+c(d t-d T)+d_{\text {orb }}+d_{\text {trop }} \\
& +d_{\text {mult }}+0.5 \lambda_{i} N_{i}+\varepsilon\left[P_{S M, L i}(n)\right]
\end{aligned}
$$

where $P_{S M}$ represents the smoothing code observations; $\lambda_{i}$ is the wavelength; $m$ is the number of observations for smoothing; and $n$ is the epoch of the observation.

Equations (3) and (4) show that the errors and noises are significantly reduced. The number of unknown parameters decreases so the convergence time can be shortened.

After IGS precise ephemerides and satellite clock data are introduced, Eqs. (3) and (4) can be rewritten as (AbdelSalam et al. 2002):

$$
\begin{aligned}
& \widetilde{P}_{S M, L i}=\rho-c d T+d_{\text {trop }}+0.5 \lambda_{i} N_{i}+\varepsilon\left[P_{S M, L i}(n)\right] \\
& \widetilde{\Phi}(n)=\rho-c d T+d_{\text {trop }}+\alpha_{1} \lambda_{1} N_{1}+\alpha_{2} \lambda_{2} N_{2}+\varepsilon[\Phi(L 1+L 2)]
\end{aligned}
$$

This model can be used to estimate the position coordinates, receiver clock errors, and ambiguities of $L 1$ and $L 2$.
The Saastamoinen model (Saastamoinen 1973) is used to correct tropospheric delay.

Four kinds of precise ephemerides and satellite clock data provided by the IGS can be classified according to the latency of release: final, rapid, observed half ultra-rapid and predicted half ultra-rapid products. The detailed description of different precise ephemerides can be found in IGS website (http://igscb.jpl.nasa.gov/).

In order to achieve positioning accuracy at the cmlevel, corrections such as relativity, satellite phase centre offset, satellite wind up, solid earth tide, ocean tide loading are applied (Héroux and Kouba 2001). In the study area, the ocean tide loading correction is relatively small at $<1 \mathrm{~cm}$ (Yeh et al. 2011) compared to an ocean tide of about $1 \mathrm{~m}$ observed by the GPS buoy.

\section{EXPERIMENT AND DATA PROCESSING}

In this study, five campaigns were conducted near the tide gauge station in Anping Harbor, Tainan, in the daytime on 9 January, 9 February, 23 February, 13 May, 14 May, and 15 May of 2009, respectively. One GPS buoy was deployed near the Anping gauge at $2-3 \mathrm{~m}$ and another GPS buoy with the same instrument was set at second-order GPS tracking station $\mathrm{S} 810$, which is $1.5 \mathrm{~km}$ away from the buoy, to obtain two simultaneous observations. For positioning precision and accuracy analyses, a series of experiments was conducted, as described in the following sections.

\subsection{Influence of Baseline Length on GPS Buoy Positioning}

The effect of baseline length on GPS positioning on land has been known well; however, it on GPS buoy positioning is checked again since the conditions of deployed buoy location are totally different. In order to analyze the influence of baseline length on the accuracy of the GPS buoy positioning, five reference stations (CISH, KASH, PKGM, WIAN, and S810) were chosen successively for the GPS buoy to facilitate DGPS kinematic positioning for two days. The relative locations of the GPS buoy and the reference stations and the distances between the buoy and the respective stations are shown in Fig. 2 and Table 1.

Since S 810 is only $1.5 \mathrm{~km}$ away from the GPS buoy, the atmospheric characteristics of the path of the signal transmission can be considered to be approximately the same. The system errors can thus be almost significantly reduced using the DGPS algorithm; therefore, the solution derived by DGPS can be considered as the true value for this experiment. For all stations, $1-\mathrm{Hz}$ dual-frequency carrier phase observations with satellite elevation larger than $15^{\circ}$ were used.

Table 2 shows the daily RMSE difference of the GPS buoy ellipsoidal heights derived using the four stations as references and the true value. The daily RMSE difference varied from 6 to $7 \mathrm{~cm}$ corresponding to the distances 
between the reference stations and the GPS buoy. When the farthest reference station from the GPS buoy is used, the two-day mean RMSE is largest.

For buoys far from reference stations for example, buoys deployed in the center of the Pacific Ocean, system errors are no longer similar to those at the reference stations, resulting in a lower accuracy derived by DGPS. Therefore, a solution by PPP that can reach the $\mathrm{cm}$ level will be more feasible for collecting data in the fields.

\subsection{Comparison of Solutions Obtained Using Kinematic PPP and DGPS Methods}

Precise ephemerides and satellite clock error data of various IGS products were utilized to calculate the GPS buoy coordinates using kinematic PPP over 5 campaigns. The results were compared to the solutions obtained with a DGPS algorithm referencing the S810 station. Because many unknown parameters have to be considered using a PPP technique, many satellites are necessary; however, low-elevation satellite signals have excessive delays, so the criteria of satellite elevation was set as $10^{\circ}$ in this study. In addition, the antenna of the GPS buoy was placed very close to sea surface to minimize multipath effects (Cheng 2005).

IGS provides four levels of precise ephemeris and satellite clock error data. The first is the final product, which has the highest accuracy and a release latency of about 13 days. The second is the rapid product, which has a lower accuracy and a release latency of 17 hours. This ephemeris is more suitable for our experiments because it can be acquired on the same day as GPS buoy observations.

The third and fourth levels are both ultra-rapid products. The former is the observed half, which is released with a latency of 3 hours, and the latter is the predicted half, which is real-time data (IGS 2002). The accuracy of the observed half is lower than that of the rapid product but higher than that of predicted half. Bar-Sever and Dow (2002) suggested that the latency for real-time processing and applications should be below one hour. However, they also mentioned that the requirements of real-time processing are too strict to achieve and suggested near-real-time (NRT) as a more flexible concept, with a latency of 0 - 6 hours.

Tables 3 - 6 show the RMSE differences of the GPS buoy E-, N-, and U-directions using a PPP technique with various IGS products and a DGPS method. The analysis indicates that the RMS differences increase with decreasing accuracy of IGS products. When using the IGS final product, the RMSE difference is $1-4 \mathrm{~cm}$ in the horizontal and $7-11 \mathrm{~cm}$ in the vertical. When using the rapid product, the RMSE difference increases to $4-7 \mathrm{~cm}$ in the horizontal and $11-18 \mathrm{~cm}$ in the vertical. The RMSE difference is on the decimeter level when the observed half is used; however, the RMSE difference increases dramatically to the meter level

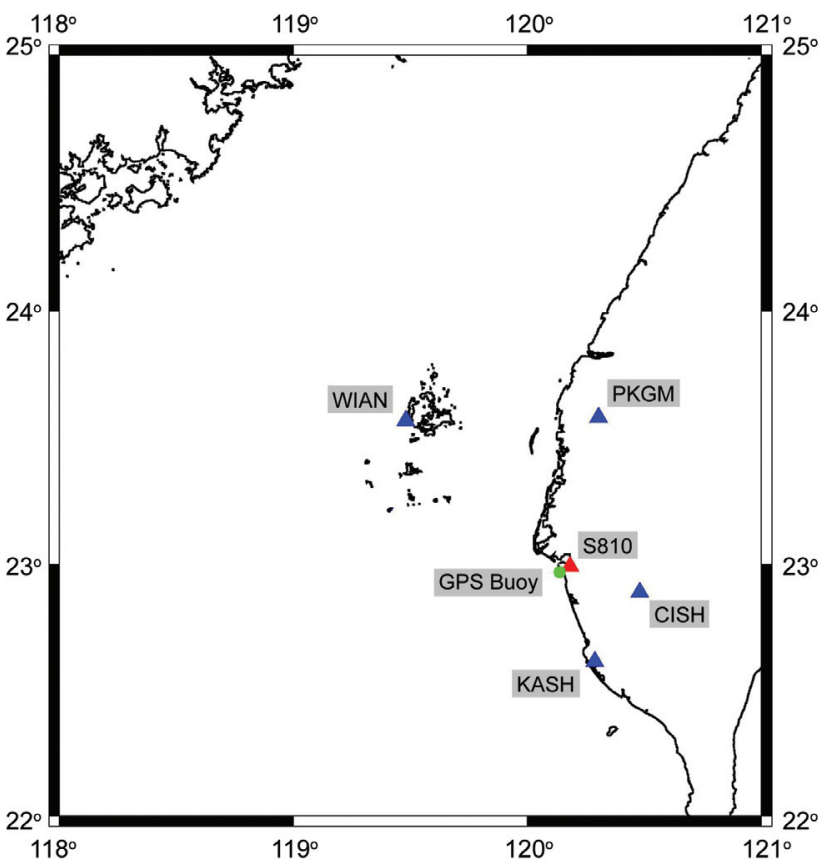

Fig. 2. Locations of 4 e-GPS satellite tracking stations, GPS buoy, and second-order GPS tracking station S810.

Table 1. Distances between the reference stations and the GPS buoy (unit: $\mathrm{km}$ ).

\begin{tabular}{c|ccccc}
\hline & S810 & CISH & KASH & PKGM & WIAN \\
\hline Distance & 1.5 & 32.8 & 42.1 & 68.2 & 96.5 \\
\hline
\end{tabular}

Table 2. Daily RMSE difference of the GPS buoy ellipsoidal heights derived by using the four stations as references (unit: meter).

\begin{tabular}{c|cccc}
\hline & CISH & KASH & PKGM & WIAN \\
\hline $2009 / 01 / 09$ & 0.086 & 0.054 & 0.054 & 0.082 \\
$2009 / 02 / 05$ & 0.035 & 0.068 & 0.075 & 0.058 \\
Mean & 0.061 & 0.061 & 0.065 & 0.070 \\
\hline
\end{tabular}

Table 3. RMSE difference of the GPS buoy E-, N-, and U-directions using PPP with IGS final product and DGPS (RMS, unit: meter).

\begin{tabular}{c|ccc}
\hline & $\mathbf{E}$ & $\mathbf{N}$ & $\mathbf{U}$ \\
\hline $2009 / 01 / 09$ & 0.015 & 0.019 & 0.111 \\
$2009 / 02 / 23$ & 0.020 & 0.029 & 0.113 \\
$2009 / 05 / 13$ & 0.045 & 0.020 & 0.106 \\
$2009 / 05 / 14$ & 0.041 & 0.022 & 0.074 \\
$2009 / 05 / 15$ & 0.024 & 0.016 & 0.079 \\
\hline
\end{tabular}


Table 4. RMSE difference of the GPS buoy E-, N-, and U-directions using PPP with IGS rapid product and DGPS (unit: meter).

\begin{tabular}{c|ccc}
\hline & $\mathbf{E}$ & $\mathbf{N}$ & $\mathbf{U}$ \\
\hline $2009 / 01 / 09$ & 0.042 & 0.044 & 0.114 \\
$2009 / 02 / 23$ & 0.065 & 0.075 & 0.180 \\
$2009 / 05 / 13$ & 0.064 & 0.046 & 0.133 \\
$2009 / 05 / 14$ & 0.043 & 0.047 & 0.110 \\
$2009 / 05 / 15$ & 0.043 & 0.040 & 0.112 \\
\hline
\end{tabular}

Table 5. RMSE difference of the GPS buoy E-, N-, and U-directions using PPP with observed half of IGS ultra-rapid product and DGPS (unit: meter).

\begin{tabular}{c|ccc}
\hline & $\mathbf{E}$ & $\mathbf{N}$ & $\mathbf{U}$ \\
\hline $2009 / 01 / 09$ & 0.171 & 0.124 & 0.380 \\
$2009 / 02 / 23$ & 0.093 & 0.104 & 0.444 \\
$2009 / 05 / 13$ & 0.272 & 0.115 & 0.357 \\
$2009 / 05 / 14$ & 0.170 & 0.110 & 0.389 \\
$2009 / 05 / 15$ & 0.114 & 0.106 & 0.238 \\
\hline
\end{tabular}

Table 6. RMSE difference of the GPS buoy E-, N-, and U-directions using PPP with predicted half of IGS ultra-rapid product and DGPS (unit: meter).

\begin{tabular}{c|ccc}
\hline & $\mathbf{E}$ & $\mathbf{N}$ & $\mathbf{U}$ \\
\hline $2009 / 01 / 09$ & 0.837 & 0.479 & 1.414 \\
$2009 / 02 / 23$ & 0.645 & 0.175 & 1.093 \\
$2009 / 05 / 13$ & 0.934 & 0.490 & 2.078 \\
$2009 / 05 / 14$ & 0.574 & 0.252 & 0.475 \\
$2009 / 05 / 15$ & 1.425 & 0.505 & 1.367 \\
\hline
\end{tabular}

when the predicted half is used. Both positioning accuracies could not satisfy the sea level monitoring criteria. In addition, we also calculated the position dilution of precision (PDOP) and the number of satellites, and the analysis shows that a large variation of PDOP and fewer satellites increase the convergence time and decrease positioning accuracy.

\subsection{Comparison Between GPS Buoy and Tide Gauge Data}

Sea level variations observed by the GPS buoy and the tide gauge were compared and results are shown in this section. 1-Hz coordinates of the GPS buoy were averaged every six minutes to match the tide gauge records and to diminish noise. In addition, in order to solve the problem of different datum, the records of the buoy and the gauge are subtracted by their own first record. The result of the analysis is shown in Table 7. The accuracy of sea level variations observed by the GPS buoy, whose coordinates are estimated by DGPS, compared to the tide gauge reaches $5 \mathrm{~cm}$. The accuracies of the solutions obtained using PPP with IGS final, rapid, observed half, and predicted half reach $5-7 \mathrm{~cm}, 7-13 \mathrm{~cm}$, decimeter level, and meter level, respectively. The correlation coefficients are $>0.95$ between tide gauge records and the positioning solutions derived by DGPS or PPP with IGS final product. Therefore, the PPP method with the IGS final product has the best accuracy and can be used for monitoring sea level changes.

The GPS buoy for these five campaigns was deployed in Anping harbor near the gauge. When the sea surface is relatively low due to a low ocean tide, the GPS signals were blocked by the breakwater. Here, we also computed the ratio of cycle slips to observations for each campaign (as shown in Table 8). The GPS buoy's ratios of cycle slips to observations are relatively lower than the S810's ratios. An unsuitable environment can lead to cycle slips, fewer acquired satellites, and a large or extremely variable PDOP, possibly reducing the positioning accuracy and increasing convergence time. Therefore, the accuracy of GPS buoys deployed in the open ocean can be further improved.

\section{ANALYSIS OF SEA LEVEL CHANGES OBSERVED BY GPS USING EMPIRICAL MODE DECOMPOSI- TION (EMD) METHOD}

The sea level time series observed by a GPS buoy using PPP and DGPS are analyzed using the EMD method. The EMD method developed by Huang et al. (1998) is used to decompose sea level signals, $x(t)$, into a series of orthogonal modes or an intrinsic mode function (IMF), $C_{i}(t)$. This method is an empirical and adaptive signal processing tool particularly suitable for non-stationary and nonlinear time series decomposition. Each IMF, $C_{i}(t)$, represents a symmetric envelope with the amplitude and frequency modulated component derived by the "sifting procedure" ( $\mathrm{Wu}$ and Huang 2009):

$x(t)=\sum_{i=1}^{n} c_{i}(t)+r_{n}$

where $r$ is the residual of the decomposition and represents a monotonic function or a trend.

All IMFs are directly derived from the signals in terms of no assumption on the linearity and stationarity. The traditional Fourier-filter, in fact, is violated by non-stationary and 
Table 7. RMSE difference (unit: meter) and correlation coefficients (CCs) of sea level variations observed by the tide gauge and the GPS buoy using using PPP with various IGS products and DGPS.

\begin{tabular}{|c|c|c|c|c|}
\hline 2009 & IGS Product & PPP-DGPS RMSE (CC) & PPP-TG RMSE (CC) & DGPS-TG RMSE (CC) \\
\hline \multirow{4}{*}{ 01/09 } & Final Product & $0.076(0.96)$ & $0.058(0.98)$ & $0.052(0.96)$ \\
\hline & Rapid Product & $0.086(0.89)$ & $0.083(0.91)$ & $0.052(0.96)$ \\
\hline & Ultra-Rapid Product (observed half) & $0.360(0.00)$ & $0.378(-0.07)$ & $0.052(0.96)$ \\
\hline & Ultra-Rapid Product (predicted half) & $2.144(0.41)$ & $2.160(0.30)$ & $0.052(0.73)$ \\
\hline \multirow{4}{*}{$02 / 23$} & Final Product & $0.070(0.70)$ & $0.061(0.83)$ & $0.047(0.73)$ \\
\hline & Rapid Product & $0.120(0.52)$ & $0.131(0.39)$ & $0.047(0.73)$ \\
\hline & Ultra-Rapid Product (observed half) & $0.158(0.46)$ & $0.174(0.33)$ & $0.047(0.73)$ \\
\hline & Ultra-Rapid Product (predicted half) & $0.661(0.14)$ & $0.644(0.37)$ & $0.047(0.73)$ \\
\hline \multirow{4}{*}{$05 / 13$} & Final Product & $0.111(0.98)$ & $0.070(0.98)$ & $0.056(0.99)$ \\
\hline & Rapid Product & $0.140(0.99)$ & $0.102(0.94)$ & $0.056(0.99)$ \\
\hline & Ultra-Rapid Product (observed half) & $0.260(0.66)$ & $0.224(0.67)$ & $0.056(0.99)$ \\
\hline & Ultra-Rapid Product (predicted half) & $2.330(-0.73)$ & $2.290(-0.72)$ & $0.056(0.99)$ \\
\hline \multirow{4}{*}{$05 / 14$} & Final Product & $0.057(0.98)$ & $0.052(0.98)$ & $0.037(0.99)$ \\
\hline & Rapid Product & $0.080(0.95)$ & $0.076(0.95)$ & $0.037(0.99)$ \\
\hline & Ultra-Rapid Product (observed half) & $0.245(0.57)$ & $0.251(0.57)$ & $0.037(0.99)$ \\
\hline & Ultra-Rapid Product (predicted half) & $0.718(0.80)$ & $0.726(0.77)$ & $0.037(0.99)$ \\
\hline \multirow{4}{*}{$05 / 15$} & Final Product & $0.058(0.83)$ & $0.051(0.86)$ & $0.028(0.96)$ \\
\hline & Rapid Product & $0.085(0.71)$ & $0.074(0.74)$ & $0.028(0.96)$ \\
\hline & Ultra-Rapid Product (observed half) & $0.131(-0.05)$ & $0.119(-0.13)$ & $0.028(0.96)$ \\
\hline & Ultra-Rapid Product (predicted half) & $0.897(-0.22)$ & $0.889(-0.29)$ & $0.028(0.96)$ \\
\hline
\end{tabular}

Table 8. GPS buoy's and S810's ratios of cycle slips to observations.

\begin{tabular}{c|ccccc}
\hline & $\mathbf{2 0 0 9 / 0 1 / 0 9}$ & $\mathbf{2 0 0 9 / 0 2 / 2 3}$ & $\mathbf{2 0 0 9 / 0 5 / 1 3}$ & $\mathbf{2 0 0 9 / 0 5 / 1 4}$ & $\mathbf{2 0 0 9 / 0 5 / 1 5}$ \\
\hline Buoy's ratio & $1: 2004$ & $1: 2054$ & $1: 1800$ & $1: 2800$ & $1: 5038$ \\
S810's ratio & $1: 225315$ & $1: 35734$ & $1: 9771$ & $1: 10271$ & $1: 8411$ \\
\hline
\end{tabular}

nonlinear processes. Thus, this method is adaptively applied as a time-frequency filter (Flandrin et al. 2004). Figure 3 shows the complete decomposition of the GPS buoy derived sea level variation on 9 January 2009 by PPP. The time series is isolated into 13 modes and a trend, and the corresponding spectrums. The high frequency modes (modes 1 - 6) are ocean waves associated with the wind-generated force and harbor seiches affected by the dimension of the harbor (So- rensen 2005). The intermediate oscillations (modes 7 - 9) can be classified as the "meteotsunamis" (Monserrat et al. 2006). The tidal components are represented in modes 10 13. The shading area in time series is the ensemble spread and the ensemble average of each IMF by the improved EMD method, Ensemble EMD (Wu et al. 2009). Consequently, GPS buoy measurements can provide more useful high-frequency ocean signals than tide gauge data; for ex- 
ample, modes 1 - 9 cannot be detected in tide gauge records with a conventional 6-minute sampling interval.

\section{DISCUSSION AND CONCLUSIONS}

In the study, a $1-\mathrm{Hz}$ geocentric sea level time series is successfully observed by a GPS buoy using the PPP tech- nique wherein the accuracy is properly assessed by comparing the tide gauge records and the solution derived by the DGPS technique. In addition, various frequency signals are obtained from the GPS buoy time series by the Ensemble EMD method. We conclude that the sea level time series derived by a GPS buoy system using the PPP technique with the final IGS product can provide high-frequency sea level
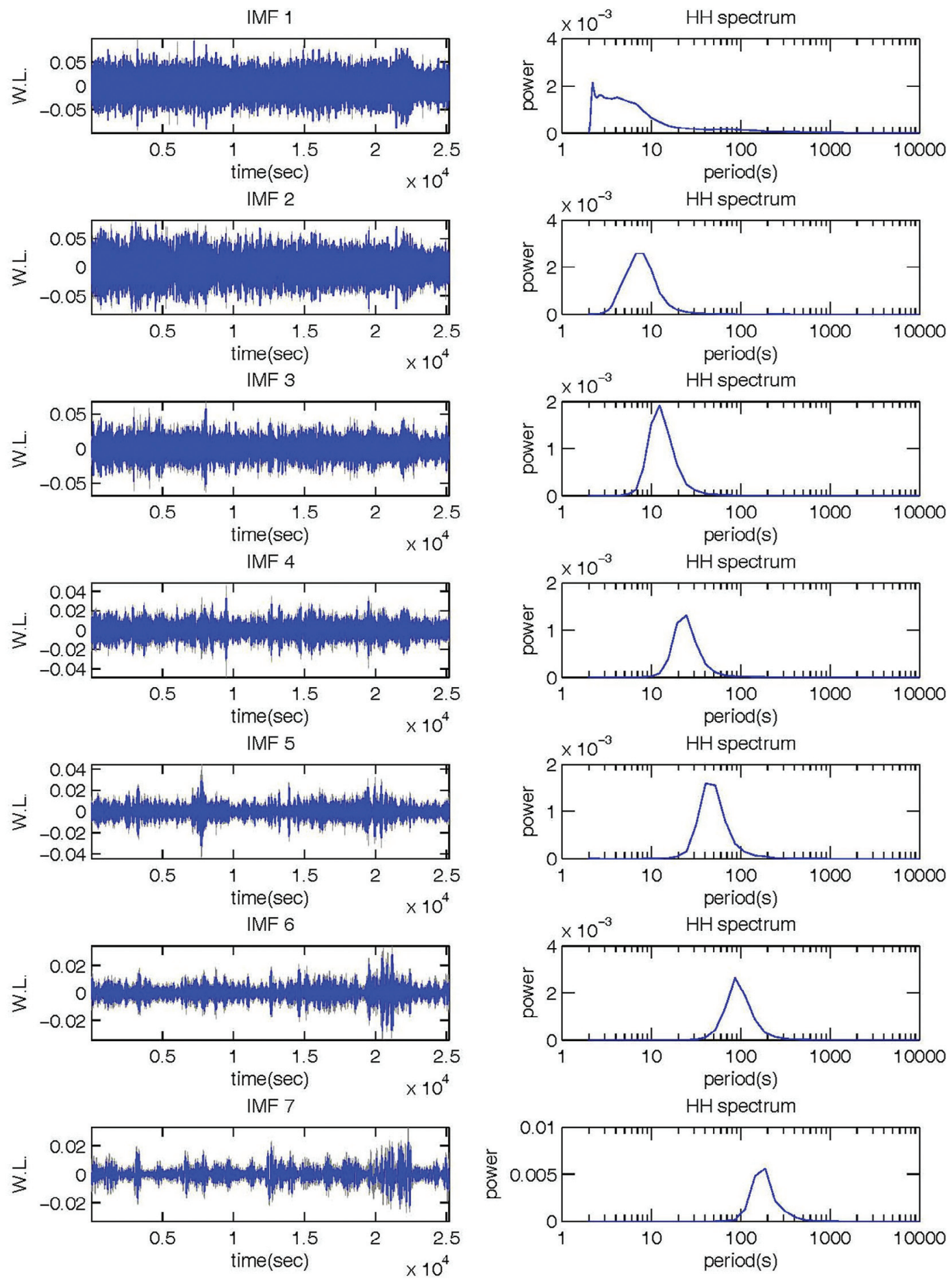

Fig. 3. The complete decomposition of the GPS buoy derived sea level variation on 9 January 2009 solved by a PPP technique using the EMD method. The left panels are 13 IMF modes and a trend. The right panels are corresponding spectrums. 

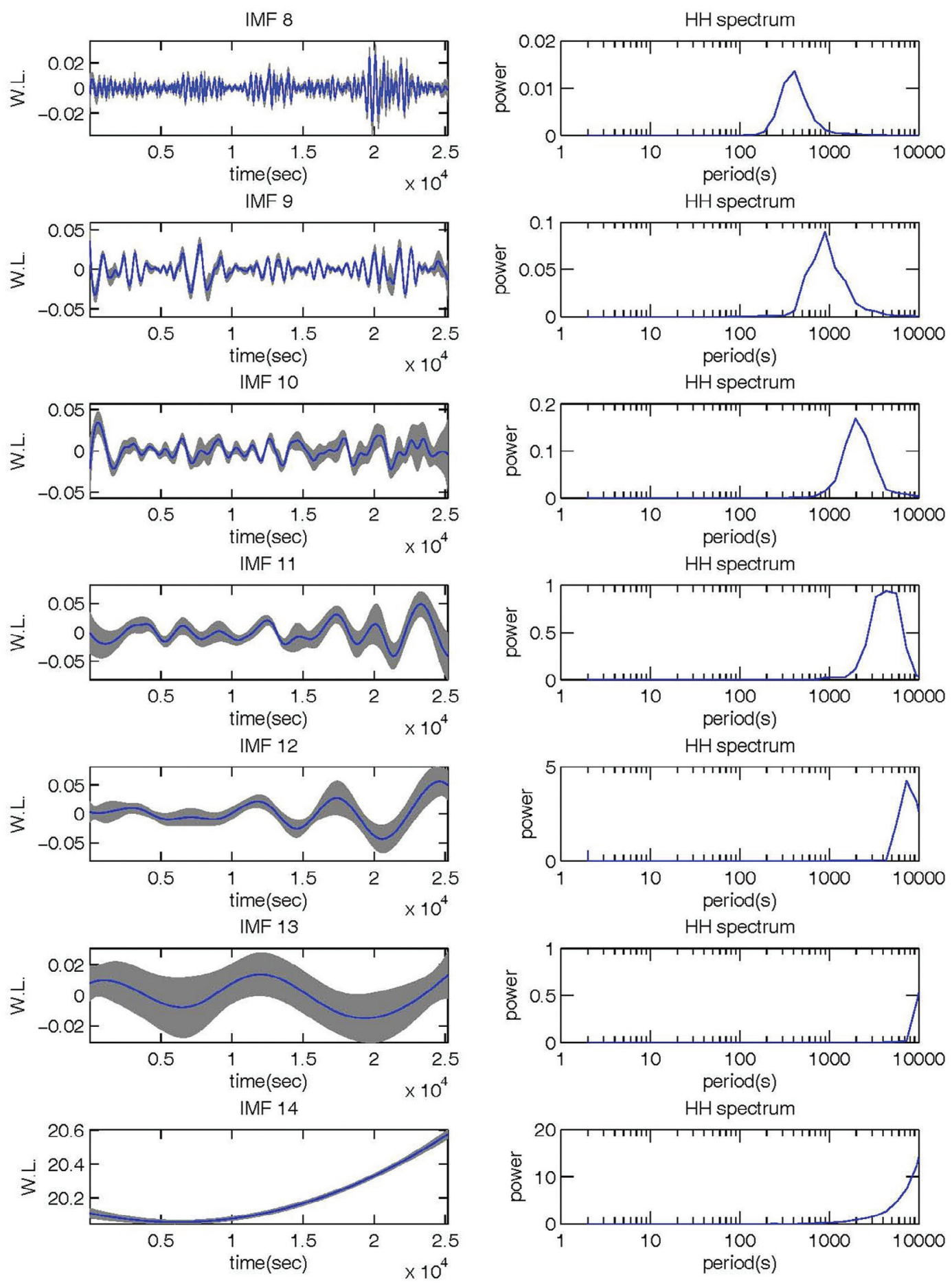

Fig. 3. (Continued)

variations with an accuracy of $5-7 \mathrm{~cm}$ that cannot be detected from 6-minute sampling tide gauge records.

The RMSE difference of the GPS buoy using PPP and DGPS is about $1-4 \mathrm{~cm}$ in the horizontal component and $7-11 \mathrm{~cm}$ in the vertical when the IGS final product is used for the kinematic PPP method. This accuracy satisfies most positioning needs for a highly varied sea surface. When the
IGS rapid product is used for PPP, the RMSE difference is $4-7 \mathrm{~cm}$ in the horizontal component and $11-18 \mathrm{~cm}$ in the vertical, which agrees with the computation provided by Chen et al. (2004). When the observed half of the IGS ultrarapid product is used, the RMSE difference is $9-27 \mathrm{~cm}$ in the horizontal component and $23-44 \mathrm{~cm}$ in the vertical. This accuracy is suitable for detecting larger sea level varia- 
tions, such as tsunamis or storm surges. When the predicted half is used, the RMSE difference is $<1 \mathrm{~m}$ in the horizontal component and $<2 \mathrm{~m}$ in the vertical, which is not ideal for sea level observations.

If the tide gauge records are considered as the true sea level variations at 6-minutes or longer in frequency, the average RMSE difference of the GPS buoy derived by DGPS is $3-5 \mathrm{~cm}$. When the IGS final, the rapid product, and the ultra-rapid products (observed and predicted) are used for the buoy derived by the PPP method, the average RMSE differences of the sea level variations are $5-7 \mathrm{~cm}, 7-13 \mathrm{~cm}$, decimeter level, and 1 - $2 \mathrm{~m}$, respectively. Therefore, sea level variations can be potentially monitored by implementing a GPS buoy using PPP with the IGS final product instead of tide gauge stations. In this study, GPS buoys were deployed in an unideal location for five campaigns, where GPS signals would be blocked by breakwater, so positioning accuracy can be improved if deployed in the open ocean to avoid cycle slips.

Traditional tide gauge records provided by data centers such as the Permanent Service for Mean Sea Level are in 6-minute to monthly sampling intervals that do not have the capability of investigating high-frequency ocean signals. Here, high-frequency GPS buoy measurements can be decomposed to obtain high-frequency ocean signals such as waves and meteotsunamis. Therefore, a GPS buoy system could provide geocentric ocean signals with different frequencies while 6-minute sampling tide gauge records affected by crustal motions do not. High-frequency ocean signals have a potential to study oceanography and improve ocean models including studying the mechanism of meteotsunamis and its causes (harbor resonance or climate).

The receivers used in the experiments do not support an L2C signal when five campaigns were performed. If using a receiver supporting an $\mathrm{L} 2 \mathrm{C}$ format with a sufficient number of satellites, the positioning accuracy should be further improved. In addition, more accurate real-time orbit and clock products, provided by the Jet Propulsion Laboratory (JPL) with a service charge, could improve the positioning accuracy as well (Chen 2004). Because the PPP process engine applied in this study does not support real time orbit and clock products at the moment; therefore, the scope of this study is limited to investigating the potential of using a PPP technique for sea level monitoring, instead of proposing a real time monitoring system. Relying on improvements in methods, the GPS buoys with a kinematic PPP technique can extend to more applications such as a tsunami forecasting system. Compared to satellite altimetry and tide gauges, mobile GPS buoys with continual high-sample measurements have the potential to monitor ocean change faithfully, especially for coastal regions.

Acknowledgements This work is supported by grants from the National Science Council of Taiwan (NSC 99-2221-E-
006-180) and National Cheng Kung University. We acknowledge the Kaohsiung Harbor Bureau for the assistance to collect GPS buoy data in the Anping Harbor. We thank two reviewers and editors for their constructive comments.

\section{REFERENCES}

Abdel-Salam, M., Y. Gao, and X. Shen, 2002: Analyzing the performance characteristics of a precise point positioning system. Proceedings of the ION GPS-2002, Oregon Convention Centre, Portland, Oregon, USA, September 24-27.

Bar-Sever, Y. and J. Dow, 2002: Position paper for the real time applications and products session. IGS Towards Real-Time Network, Data, Analysis Center 2002 Workshop, Ottawa, Canada, April 8-11.

Cardellach, E., D. Behrend, G. Ruffini, and A. Rius, 2000: The use of GPS buoys in the determination of oceanic variables. Earth Planets Space, 52, 1113-1116.

Chen, K., 2004: Real-time precise point positioning and its potential applications. Proceedings of the $17^{\text {th }}$ International Technical Meeting of the Satellite Division of The Institute of Navigation (ION GNSS 2004), Long Beach, CA, September 2004, 1844-1854.

Chen, W., C. Hu, Z. Li, Y. Chen, X. Ding, S. Gao, and S. Ji, 2004: Kinematic GPS precise point positioning for sea level monitoring with GPS buoy. JGPS, 3, 302-307.

Cheng, K. C., 2005: Analysis of water level measurements using GPS. Ph.D. Dissertation, The Ohio State University, Columbus, Ohio, USA.

Cheng, K. C., C. Y. Kuo, C. K. Shum, X. Niu, R. Li, and K. Bedford, 2008: Accurate linking of Lake Erie water level with shoreline datum using GPS buoy and satellite altimetry. Terr. Atmos. Ocean. Sci., 19, 53-62, doi: 10.3319/TAO.2008.19.1-2.53(SA). [Link]

Cheng, K. C., S. Calmant, C. Y. Kuo, H. Z. Tseng, C. K. Shum, F. Seyler, and J. S. D. Silva, 2009: Branco river stage gradient determination and Amazon hydrologic studies using GPS Water level measurements. Mar. Geodesy, 32, 267-283, doi: 10.1080/01490410903094 460. [Link]

Cheng, K. C., C. Y. Kuo, H. Z. Tseng, Y. Yi, and C. K. Shum, 2010: Lake surface height calibration of Jason-1 and Jason-2 over the Great Lakes. Mar. Geodesy, 33, 186-203, dio: 10.1080/01490419.2010.487802. [Link]

Del Cogliano, D., R. Dietrich, A. Richter, R. Perdomo, J. L. Hormaechea, G. Liebsch, and M. Fritsche, 2007: Regional geoid determination in Tierra del Fuego including GPS levelling. Geol. Acta, 5, 315-322.

Flandrin, P., G. Rilling, and P. Goncalves, 2004: Empirical mode decomposition as a filter bank. IEEE Signal Process. Lett., 11, 112-114, doi: 10.1109/LSP.2003.82 1662. [Link] 
Frappart, F., S. Calmant, M. Cauhopé, F. Seyler, and A. Cazenave, 2006: Preliminary results of ENVISAT RA-2derived water levels validation over the Amazon Basin. Remote Sens. Environ., 100, 252-264, doi: 10.1016/j. rse.2005.10.027. [Link]

Gao, Y., M. Abdel-Salam, K. Chen, and A. Wojciechowski, 2003: Point real-time kinematic positioning. Proceedings of the International Association of Geodesy IAG General Assembly Sapporo, Japan, June 30 - July 11.

Héroux, P. and J. Kouba, 2001: GPS precise point positioning using IGS orbit products. Phys. Chem. Earth A, 26, 573-578, doi: 10.1016/S1464-1895(01)00103-X. [Link]

Héroux, P., J. Kouba, P. Collins, and F. Lahaye, 2001: GPS carrier-phase point positioning with precise orbit products. Proceedings of the KIS 2001, Banff, Alberta, Canada, June 5-8.

Huang, N. E. and Z. Wu, 2008: A review on Hilbert-Huang transform: Method and its applications to geophysical studies. Rev. Geophys., 46, RG2006, doi: 10.1029/20 07RG000228. [Link]

Huang, N. E., Z. Shen, S. R. Long, M. C. Wu, H. H. Shih, Q.Zheng, N. C. Yen, C. C. Tung, and H. H. Liu, 1998: The empirical mode decomposition and the Hilbert spectrum for nonlinear and non-stationary time series analysis. Proc. R. Soc. Lond. A, 454, 903-995, doi: 10. 1098/rspa.1998.0193. [Link]

IGS (International GNSS Service), 2002, available at http:// igscb.jpl.nasa.gov/.

Lee, H., C. K. Shum, W. Emery, S. Calmant, X. Deng, C. Y. Kuo, C. Roesler, and Y. Yi, 2010: Validation of Jason-2 altimeter data by waveform retracking over California coastal ocean. Mar. Geodesy, 33, 304-316, doi: 10.1080/01490419.2010.488982. [Link]

Li, X., X. Zhang, and F. Guo, 2009: Study on precise point positioning based on combined GPS and GLONASS. Proceedings of the $22^{\text {nd }}$ International Technical Meet- ing of The Satellite Division of the Institute of Navigation (ION GNSS 2009), Savannah, GA, September 2009, 2449-2459.

Monserrat, S., I. Vilibić, and A. B. Rabinovich, 2006: Meteotsunamis: Atmospherically induced destructive ocean waves in the tsunami frequency band. Nat. Hazards Earth Syst. Sci., 6, 1035-1051, doi: 10.5194/nhess -6-1035-2006. [Link]

Saastamoinen, J., 1973: Contributions to the theory of atmospheric refraction. Part II. Refraction corrections in satellite geodesy. Bulletin Géodésique, 107, 13-34, doi: 10.1007/BF02522083. [Link]

Sorensen, R. M., 2005: Basic Coastal Engineering. $3^{\text {rd }}$ edition, Springer, New York.

Willis, J. K., D. P. Chambers, C. Y. Kuo, and C. K. Shum, 2010: Global sea level rise: Recent progress and challenges for the decade to come. Oceanography, 23, 2635.

Wu, Z. and N. E. Huang, 2009: Ensemble empirical mode decomposition: A noise-assisted data analysis method. AADA, 1, 1-41, doi: 10.1142/S1793536909000047. [Link]

Yeh, T. K., C. Hwang, J. F. Huang, B. F. Chao, and M. H. Chang, 2011: Vertical displacement due to ocean tidal loading around Taiwan based on GPS observations. Terr. Atmos. Ocean. Sci., 22, 373-382, doi: 10.3319/ TAO.2011.01.27.01(T). [Link]

Zhang, X. and O. B. Andersen, 2006: Surface ice flow velocity and tide retrieval of the Amery ice shelf using precise point positioning. J. Geodesy, 80, 171-176, doi: 10.1007/s00190-006-0062-8. [Link]

Zumberge, J. F., M. B. Heflin, D. C. Jefferson, M. M. Watkins, and F. H. Webb, 1997: Precise point positioning for the efficient and robust analysis of GPS data from large networks. J. Geophys. Res., 102, 5005-5017, doi: 10.1029/96JB03860. [Link] 